Memoirs of the College of Science, University of Kyoto, Series A,

Vol. XXVI, Mathematics No. 3, 1951.

\title{
New Treatise of Solutions of a System of Ordinary Differential Equations and its Application to the Uniqueness Theorems.
}

By

\author{
Kyuzo HAYASHI and Taro Yoshizawa
}

(Received December 20, 1950)

\section{§ 1. Introduction.}

To explain simply let us consider the differential equation $\frac{d y}{d x}=f(x, y)$ defined in a domain of two dimensions. To each point $(x, y)$ of the domain we adjoin the direction $f(x, y)$; so we have a manifoldness of points and directions. To solve the differential equation is nothing but to find a locus of point and direction in the manifoldness such that, for all $(x, y), f(x, y)$ shall be equal to $\frac{d y}{d x}$. Therefore we consider a curve $y=\varphi(x)$ say in the domain; then its $(x, y)$ and $\varphi^{\prime}(x)$ construct a locus, generally different from a locus in the manifoldness. Then at each point on the curve $y=\varphi(x)$, the deviation of its direction $\varphi^{\prime}(x)$ from that of the manifoldness is given by $\left|\varphi^{\prime}(x)-f(x, \varphi(x))\right|$. Along the curve $y=\varphi(x)$ we sum up the deviations, i. e., the totaldeviation is $\int\left|\varphi^{\prime}(x)-f(x, \varphi(x))\right| d x$. Such quantity is considered in statistics. If it be zero, $y=\varphi(x)$ is possibly a solution of the differential equation. Thus we arrive at the idea given below (2). We remark that naturally we may define many other measures of deviations.

In this paper we shall perform integrating operations always in the sense of Lebesgue.

Consider a system of ordinary differential equations 


$$
\frac{d y_{i}}{d x}=f_{i}\left(x, y_{1}, y_{i}, \ldots, y_{n}\right) \quad(i=1,2, \ldots, n),
$$

where $f_{i}\left(x, y_{1} y_{2}, \ldots, y_{n}\right)$ are defined in a domain ${ }^{(1)}$

$$
G: 0 \leqq x \leqq a,\left|y_{i}\right| \leqq b_{i}(i=1,2, \ldots, n),
$$

having the properties as follows:

a) they are measurable with regard to $x$, and continuous functions of $\left(y_{1} y_{2}, \ldots, y_{n}\right)$,

b) $\left|f_{i}\left(x, y_{1}, y_{2}, \ldots y_{n}\right)\right| \leqq M_{i}(x) \quad(i=1,2, \ldots, n)$, where $M_{i}(x) \quad(i=$ $1,2, \ldots, n)$ are summable, i. e. integrable in the sense of Lebesgue, for $0 \leqq x \leqq a$. For this differential system we call such functions $\varphi_{i}(x) \quad(i=1,2, \ldots, n)$ the solution passing through the point $P\left(x_{p}\right.$, $\left.y_{1,}, y_{2 p}, \ldots, y_{n_{p}}\right) \in G$ that

c) they are defined in an interval $I$ containing $x_{p}, \varphi_{i}\left(x_{p}\right)=y_{i p}$ and $\left(x, \varphi_{1}(x), \varphi_{2}(x), \ldots \ldots, \varphi_{n}(x)\right) \in G(x \in I)$,

d) $\varphi_{i}(x)=y_{i p}+\int_{x_{\nu}}^{e} f_{i}\left(x, \varphi_{1}(x), \varphi_{2}(x), \ldots, \varphi_{n}(x)\right) d x(x \in I)$.

By d) the solutions of (1) are all absolutely continuous.

Moreover we represent the vectors in the space of $n$ dimensions by $\boldsymbol{y}:$ namely $\boldsymbol{y}=\left(y_{1}, y_{2}, \ldots, y_{n}\right), \quad|\boldsymbol{y}|=\sqrt{\boldsymbol{y}_{1}^{2}+y_{z}^{2}+\ldots+y_{n}^{2}}$ Therefore (1) may be represented by

$$
\frac{d ! y}{d x}=f(x, y) \quad[(x, y) \in G] .
$$

Let us now enunciate the Carathéodory's existence theorem ${ }^{(2)}$ of solutions of ordinary differential equations, which plays an important part in this paper.

Lemma. If $\varphi(x)$ is measurable in $0 \leqq x \leqq a$ ond $\left|\varphi_{i}(x)\right| \leqq b_{i}$ $(i=1,2, \ldots, n), f(x, \varphi(x))$ is summable in $0 \leqq x \leqq a$.

The proof is omitted.

Theorem. When, in an interval $I$ containing $x_{m}$,

$$
\left(x, \boldsymbol{y}_{p} \pm \int_{x_{p}}^{x} \boldsymbol{H}(x) d x\right) \in G(x \in I),
$$

then there exists a solution of (1), which is defined in $I$ and passing through the point $P\left(x_{p}, y_{y}\right)$. Hence, if $\left|y_{i p}\right|<b_{i}(i=1,2, \ldots n)$, there exists evidently a solution of (1), which is defin $\leq d$ in a certain small interval $I$ containing $x_{t}$, and passing through the point $P$.

For the proof we refer to Carathéodory, loc. cit.. 
$\S 2$. Definition of $D(P, Q)$.

Consider two points $P=\left(x_{p}, \boldsymbol{y}_{\ell}\right)$ and $Q=\left(x_{q}, \boldsymbol{y}_{Q}\right)$ in $G$, where $x_{P}<x_{q}$. By $\mathfrak{Y}_{P Q}$ we represent the family of all such functions that are absolutely continuous in $x_{p^{\prime}} \leqq x \leqq x_{Q}$ and

$$
\boldsymbol{y}\left(x_{P}\right)=\boldsymbol{y}_{P}, \boldsymbol{y}\left(x_{Q}\right)=\boldsymbol{y}_{Q}, \quad(x, y(x)) \epsilon G\left(x_{p^{\prime}} \leqq x \leqq x_{Q_{2}}\right) .
$$

Therefore if $\boldsymbol{y}(x) \in \mathfrak{Y}_{P Q}$, its derivative $\boldsymbol{y}^{\prime}(x)$ is summable in $x_{P} \leqq x \leqq x_{Q}$. And so we can define the function $D(P, Q)$ of $P$ and $Q$ as follows; namely

$$
D(P, Q)=\inf _{\boldsymbol{y}(x) \in \mathfrak{D}^{\prime} Q} \int_{x_{P}}^{x_{Q}}\left|y^{\prime}(x)-f(x, y(x))\right| d x,^{(3)}
$$

moreover if $x_{P}=x_{Q}, D(P, Q)=\left|\boldsymbol{y}_{1}-\boldsymbol{y}_{Q}\right|^{(4)}$ and if $x_{P}>x_{Q}, D(P, Q)$ $=D(Q, P)$.

Then we have the following fundamental theorem.

Theorem. In order that the ivo points $P=\left(x_{P}, \boldsymbol{y}_{P}\right)$ and $Q=$ $\left(x_{Q}, \boldsymbol{y}_{Q}\right)$ in $G$ should lie on a same soluiion of $(1)$, it is necessary and sufficient that $D(P, Q)=0$.

Proof. Evidently the condition is necessary. Now let $D(P$, $Q)=0$, then there is a sequence of functions $\left\{\boldsymbol{y}_{\nu}(x)\right\}\left(\boldsymbol{y}(x) \in \mathfrak{Y}_{P^{\prime} \mathcal{Q}}\right.$, $\nu=1,2, \ldots)$ such as

$$
\lim _{\nu \rightarrow \infty} \int_{x_{\nu}}^{x_{Q}}\left|\boldsymbol{y}_{\nu}^{\prime}(x)-f\left(x, \boldsymbol{y}_{\nu}(x)\right)\right| d x=0 .
$$

Hence, if wa put

$$
\boldsymbol{y}_{\nu}(x)-\boldsymbol{y}_{1}-\int_{v^{\prime}}^{c} f\left(x, \boldsymbol{y}_{\nu}(x)\right) d x=\sigma_{\nu}(x)
$$

we have evidently

$$
\lim _{\nu \rightarrow \infty} \sigma_{\nu}(x)=0 \quad\left(i=1,2, \ldots, n, x_{1} \leqq x \leqq x_{\imath}\right)
$$

Put

$$
\boldsymbol{Y}_{\nu}(x)=\boldsymbol{y}_{\nu}(x)-\sigma_{\nu}(x),
$$

and then, for $x_{P} \leqq x_{1} \leqq x_{2} \leqq x_{Q}$,

$$
\boldsymbol{Y}_{\nu}\left(x_{2}\right)-\boldsymbol{Y}_{\nu}\left(x_{1}\right)=\int_{x_{1}}^{x_{2}} \boldsymbol{f}\left(x, \boldsymbol{y}_{\nu}(x)\right) d x
$$


and so we have

$$
\left|\boldsymbol{Y}_{\nu}\left(x_{2}\right)-\boldsymbol{Y}_{\nu}\left(x_{1}\right)\right| \leqq \int_{x_{1}}^{c_{2}}|\boldsymbol{M}(x)| d x .
$$

Consequently the sequence of the functions $\boldsymbol{Y}_{2}(x)$ is equally continuous. Hence we can select a uniformly convergent sequence by Ascoli and Arzéla theorem, and we have in the limit

$$
\begin{gathered}
\boldsymbol{Y}\left(x_{p^{\prime}}\right)=\boldsymbol{y}_{P}, \boldsymbol{Y}\left(x_{Q}\right)=\boldsymbol{y}_{Q}, \\
\boldsymbol{Y}(\boldsymbol{x})=\boldsymbol{y}_{p^{p}}+\int_{x_{P}}^{x} \boldsymbol{f}(x, \boldsymbol{Y}(x)) d x .
\end{gathered}
$$

Therefore we have obtained a solution $\boldsymbol{y}=\boldsymbol{Y}(x)$ of (1), passing through $P$ and $Q$. Q.E. D.

Remark. Let $H_{\alpha}$ be a hyperplane by $x=u(0 \leqq \mu \leqq a)$ in $G$, and $S_{\alpha}$ an arbitrary closed sub-set in $H_{\alpha}$. Now let $S_{\xi}$ and $S_{\xi^{\prime}}$ be two closed sub-sets in $G$ such as $x=\hat{\xi}$ and $x=\hat{\xi}^{\prime}$ respectively. Then define

$$
\begin{aligned}
D\left(S_{\xi}, S_{\xi^{\prime}}\right)= & \min D(P, Q) . \\
& P \in S_{\xi} \\
& Q \in S_{\xi^{\prime}}
\end{aligned}
$$

$D\left(S_{\xi}, S_{\xi^{\prime}}\right)$ has the same properties as $D(P, Q)$; e.g., for that a solution of (1) shall exist so as to pass through $S_{\xi}$ and $S_{\xi}$, it is necessary and sufficient that $D\left(S_{\xi}, S_{\xi^{\prime}}\right)=0$.

\section{$\S$ 3. Properties of $D(P, Q)$.}

In this paragraph we shall enumerate certain important properties of $D(P, Q)$.

1) Consider two points $P$ and $Q$ in $G$ such as $x_{P}<x_{Q}$. For $\boldsymbol{y}(x) \in \mathfrak{V}^{\prime \prime}$,

$$
\begin{aligned}
& \int_{x_{p},}^{x_{Q}}\left|\boldsymbol{y}^{\prime}(x)-f(x, y(x))\right| d x \geqq \int_{x_{p}}^{x_{Q}}\left|\boldsymbol{y}^{\prime}(x)\right| d x-\int_{x_{P}}^{r_{Q}}|\boldsymbol{f}(x, \boldsymbol{y}(x))| d x . \\
& \geqq \int_{x P}^{x^{2}}\left|\boldsymbol{y}^{\prime}(x)\right| d x-\int_{x, P}^{x_{Q}}|\boldsymbol{M}(x)| d x \\
& \geqq\left|\int_{x_{p}, p}^{x_{2}} \boldsymbol{y}^{\prime}(x) \dot{d} x\right|-\int_{c P}^{x_{Q}}|\boldsymbol{M}(x)| d x^{(E)} \\
& \geq\left|\boldsymbol{y}_{Q}-\boldsymbol{y}_{p}\right|-\int_{q_{p}}^{x_{p}}|\boldsymbol{M}(\boldsymbol{x})| d x \text {. }
\end{aligned}
$$


Therefore evidently we have

$$
D(P, Q) \geqq\left|\boldsymbol{y}_{Q}-\boldsymbol{y}_{P}\right|-\int_{x_{P}}^{x_{Q}}|\boldsymbol{M}(x)| d x .
$$

Now consider the function $\boldsymbol{y}(x)$ which represents the segment $\overline{P Q}$, then clearly $\boldsymbol{y}(x)$ belongs to $)_{1: Q}$ and

$$
\int_{x_{P}}^{x_{Q}}\left|\boldsymbol{y}^{\prime}(x)\right| d x=1 \int_{x_{p}}^{x_{Q}} \boldsymbol{y}^{\prime}(x) d x|=| \boldsymbol{y}_{p_{2}}-\boldsymbol{y}_{p} \mid .
$$

Hence we have

$$
D(P, Q) \leqq\left|\boldsymbol{y}_{Q}-\boldsymbol{y}_{P}\right|+\int_{r_{1},}^{x_{Q}}|\boldsymbol{M}(x)| d x ;
$$

for $\int_{x_{P}}^{x_{Q}}\left|\boldsymbol{y}^{\prime}(x)-\boldsymbol{f}^{\prime}(x, \boldsymbol{y}(x))\right| d x \leqq \int_{x_{p}}^{x_{Q}}\left|\boldsymbol{y}^{\prime}(x)\right| d x+\int_{x_{p}}^{x_{y}}|\boldsymbol{M r}(x)| d x$.

Therefore if $x_{P}=x_{Q}$, it is convenient to define $D(P, Q)=\left|\boldsymbol{y}_{Q}-\boldsymbol{y}_{r}\right|$.

2) For three points $P, Q$ and $R$ such as $x_{r} \leqq x_{Q} \leqq x_{R}$,

$$
D(P, R) \leqq D(P, Q)+D(Q, R) .
$$

This is clear when $x_{i}<x_{Q}<x_{k}$. Hence let us e.g. consider the case where $x_{R},<x_{Q}=x_{R}$. Then by the definition of $D(P, Q)$, it is evidently possible to find a sequence of functions $\left\{\boldsymbol{y}_{\nu}(x)\right\} \quad\left(\boldsymbol{y}_{\nu}(x)\right.$ $\left.\epsilon \mathfrak{V}_{r Q}, \nu=1,2, \ldots\right)$ such that

$$
D(P, Q)=\lim _{\nu \rightarrow \infty} \int_{x_{\nu^{\prime}}}^{x_{\ell^{\prime}}}\left|\boldsymbol{y}_{\nu}^{\prime}(x)-f\left(x, \boldsymbol{y}_{\nu}(x)\right)\right| d x .
$$

And so take a point $Q_{\nu}=\left(x_{Q^{\prime}}, \boldsymbol{y}_{\nu}\left(x_{Q^{\prime}}\right)\right)$ such as $x_{P}<x_{Q^{\prime}}<x_{Q}=x_{R}$, then

$$
\begin{aligned}
\int_{x_{\nu}}^{x_{Q}}\left|\boldsymbol{y}_{\nu}^{\prime}(x)-f\left(x, \boldsymbol{y}_{\nu}(x)\right)\right| d x=\int_{x_{\nu^{\prime}}}^{x_{Q^{\prime}}}\left|\boldsymbol{y}_{\nu}^{\prime}(x)-\boldsymbol{f}\left(x, \boldsymbol{y}_{\nu}(x)\right)\right| d x \\
+\int_{x Q^{\prime}}^{x_{\nu^{\prime}}}\left|\boldsymbol{y}_{\nu}^{\prime}(x)-f\left(x, \boldsymbol{y}_{\nu}(x)\right)\right| d x \geqq D\left(P, Q_{\nu}\right)+D\left(Q_{\nu}, Q\right),
\end{aligned}
$$

hence for $x_{P^{\prime}}<x_{\ell^{\prime}}<x_{k}$,

$$
\begin{aligned}
\geqq D(P, R)-D\left(Q_{v}, R\right)+D\left(Q_{v}, Q\right) \\
\geqq D(P, R)-\left|\boldsymbol{y}_{P}-\boldsymbol{y}_{Q_{v}}\right|-\int_{\nu_{Q^{\prime}}}^{x_{Q}}|\boldsymbol{M}(x)| d x+\left|\boldsymbol{y}_{\iota^{\prime}}-\boldsymbol{y}_{Q_{v}}\right| \\
\quad-\int_{x_{Q^{\prime}}}^{x_{Q_{2}}}|\boldsymbol{M}(x)| d x
\end{aligned}
$$




$$
\geq D(P, R)-\left|\boldsymbol{y}_{K}-\boldsymbol{y}_{Q}\right|-2 \int_{{ }_{Q_{Q^{\prime}}}^{x}}^{x_{Q}}|\boldsymbol{M} \boldsymbol{\Gamma}(x)| d x .
$$

By tending $\nu$ to infinity, we have

$$
D(P, Q) \geqq D(P, R)-\left|\boldsymbol{y}_{R}-\boldsymbol{y}_{Q}\right|
$$

namely

$$
D(P, R) \leqq D(P, Q)+D(Q, R)
$$

for $x_{Q}$ may be arbitrarily near to $x_{\vartheta}$.

3) For three points $P, Q$ and $R$ such as $x_{R^{\prime}} \leqq x_{Q} \leqq x_{k}$, we have

(6) $\left\{\begin{array}{l}D(P, R) \geq D(P, Q)-\left|y_{R}-y_{Q}\right|-\int_{x_{Q}}^{x_{R}}|\boldsymbol{M}(x)| d x \\ D(P, R) \geqq D(\ddot{Q}, R)-\left|y_{Q}-y_{P}\right|-\int_{x_{P}}^{x_{Q}}|\boldsymbol{M}(x)| d x .\end{array}\right.$

As in 2), for $x_{P}<x_{Q}<x_{k}$, consider a sequence of functions $\left\{\boldsymbol{y}_{\llcorner}(x)\right\}$ such that

$$
D(P, R)=\lim _{\nu \rightarrow \infty} \int_{x_{P}}^{r}\left|\boldsymbol{y}_{\nu}^{\prime}(x)-f\left(x, y_{\nu}(x)\right)\right| d x
$$

and points $Q_{\nu}=\left(x_{\imath}, \boldsymbol{y}_{\nu}\left(x_{\imath}\right)\right)$. Then

$$
\begin{aligned}
& \int_{x p}^{{ }^{c} \boldsymbol{K}}\left|\boldsymbol{y}_{\nu}^{\prime}(x)-f\left(x, \boldsymbol{y}_{2}(x)\right)\right| d x=\int_{x_{p}}^{x_{Q}}\left|\boldsymbol{y}^{\prime}(x)-f\left(x, \boldsymbol{y}_{\nu}(x)\right)\right| d x \\
& +\int_{{ }^{c} c_{Q}}^{c_{R}}\left|\boldsymbol{y}_{\nu}^{\prime}(x)-f\left(x, \boldsymbol{y}_{\nu}(x)\right)\right| d x \\
& \geq D\left(P, Q_{\nu}\right)+D\left(Q_{\nu}, R\right) \\
& \geq D(P, Q)-D\left(Q_{\nu}, Q\right)+D\left(Q_{\nu}, R\right) \\
& \geq D(P, Q)-\left|\boldsymbol{y}_{Q_{\nu}}-\boldsymbol{y}_{Q}\right|+\left|\boldsymbol{y}_{R}-\boldsymbol{y}_{Q_{\nu}}\right|-\int_{x_{Q}}^{x_{R}}|\boldsymbol{H}(x)| d x \\
& \geq D(P, Q)-\left|\boldsymbol{y}_{\ell}-\boldsymbol{y}_{Q}\right|-\int_{x_{Q}}^{x_{R}}|\boldsymbol{M}(x)| d x \text {. }
\end{aligned}
$$

Hence evidently, for $x_{r} \leqq x_{Q} \leqq x_{k}$, we find (6). Consequently, in genoral; we have 


$$
|D(P, Q)-D(P, R)| \leqq\left|\boldsymbol{y}_{Q}-\boldsymbol{y}_{k}\right|+\left|\int_{x_{Q}}^{x_{k}} M(x) d x\right|,
$$

where $M(x)=|\boldsymbol{M}(x)|=\sqrt{M_{1}^{2}+M_{2}^{2} \ldots+M_{m}^{2}}$.

Then we can conclude that $D(P, Q)$ is a non-n gative continuous funtion of $(P, Q)$.

\section{§4. Lemma.}

Now let $P$ be a fixed point in $G$ and put

$$
\varphi(x, \boldsymbol{y})=D(P, Q) \quad \text { with } Q=(x, !),
$$

then, in $G, \varphi(x, y)$ is a non-negative continuous function of $(x, y)$ and, for two points $\left(x_{1}, y_{1}\right)$ and $\left(x_{2} . y_{2}\right)$, we have

(8) $\left|\varphi\left(x_{1}, \boldsymbol{y}_{1}\right)-\varphi\left(x_{2}, \boldsymbol{y}_{2}\right)\right| \leqq\left|\boldsymbol{y}_{1}-\boldsymbol{y}_{2}\right|+\left|\int_{x_{1}}^{x_{2}} M(x) d x\right|$.

If the points $Q$ and $R$ are on the same solution of (1) and $x_{p} \leqq x_{\ell} \leqq x_{k}$, then since

$$
D(P, R) \leqq D(P, Q)+D(Q, R)=D(P, Q) \quad(\text { by } D(Q, R)=0),
$$

we have

$$
\varlimsup_{t \rightarrow 0} \frac{1}{t}\left\{\varphi\left(x+t, y+\int_{x}^{x+t} f(t, \bar{y}(t)) d t\right)-\varphi(x, y)\right\} \leqq 0,
$$

$\boldsymbol{y}=\overline{\boldsymbol{y}}(x)$ being a solution passing through the point $(x, y)$. Moreover, by $(8)$,

$$
\begin{aligned}
& \left|\frac{1}{t}\left\{\varphi[x+t, \bar{y}(x+t)]-\varphi\left[x+t, y+t f^{\prime}(x, y)\right]\right\}\right| . \\
& \leqq\left|\frac{1}{t}\{\bar{y}(x+t)-y\}-f(x, y)\right|
\end{aligned}
$$

On the other hand, at almost every point in $x_{P} \leqq x \leqq a$,

$$
\lim _{t \rightarrow 0} \frac{1}{t}\{\bar{y}(x+t)-\boldsymbol{y}\}=f(x, y) \quad a . e .^{(6)} .
$$

Therefore we have, for almost every point in $x \leqslant x \leqq a$, 


$$
\varlimsup_{t \rightarrow 0} \frac{1}{t}\{\varphi(x+t, y+t f(x, y))-\varphi(x, y)\} \leqq 0 \text { a.e.. }
$$

Conversely, if there exists a function $\varphi(x, y)$ which satisfies the conditions (8) and (9), we sea that by (8) $\varphi(x, \vec{y}(x))$ is absolutely continuous, where $\boldsymbol{y}=\overline{\boldsymbol{y}}(x)$ is a solution of (1) passing through the point $(x, y) \in G(x, \leqq x)$, and by (9), for almost every point in $x_{P} \leqq x \leqq a$,

$$
\lim _{t \rightarrow 0} \frac{1}{t}\{\varphi(x+t, \bar{y}(x+t))-\varphi(x, y)\} \leqq 0 \text { a.e. }
$$

Hence $\varphi(x, y)$ does not increase with $x$ on any solution of (1).

Remark. ${ }^{(i)}$ If $f(x, y)$ is a continuous function of $(x, y)$ in $G$, the conditions (8) and (9) can be replaced respectively by the next ones:

$$
\begin{aligned}
&\left|\varphi\left(x, y_{1}\right)-\varphi\left(x, y_{2}\right)\right|\left|y_{1}-y_{2}\right| \\
& \varlimsup_{t \rightarrow 0} \frac{1}{t}\left\{\varphi\left(x+t, y+t f^{\prime}(x, y)\right)-\varphi(x, y)\right\} \leqq 0 \\
&\text { (for all } \left.x \text { in } x_{P} \leqq x \leqq a\right) .
\end{aligned}
$$

\section{§5. Applications to the uniqueness theorems.}

Thus we have succeeded to find $D(P, Q)$ which has the almost same properties as those in the case where $f(x, y)$ is continuous $(i)$ in $G$, and so we can extend, without any great modifications, the Okamura's uniqueness theorems ${ }^{(i)}$ of solutions of Cauchy-problem and our uniqueness theorems ${ }^{(s)}$ of solutions of boundary-problems. For example,

Theorem. Consider the differential system (1), where $f(x$, $\boldsymbol{O})=0(\boldsymbol{O}=(o, o, \ldots o))$ for almost every $x$ in $o \leqq x \leqq a$. Then in order that the solution of (1) passing through the origin $O(o, O)$ should be uniquz, it is necessary and sufficient that there exists a cotinuous function $\varphi(x, y)$ in $G$, such that

$$
\begin{aligned}
& \varphi(x, \boldsymbol{O})=0 \text { for } 0 \leqq x \leqq a, \\
& \varphi(x, \boldsymbol{y})>0 . \text { for } \boldsymbol{y} \neq \boldsymbol{O},
\end{aligned}
$$




$$
\left|\varphi\left(x_{1}, \boldsymbol{y}_{1}\right)-\varphi\left(y_{2}, \boldsymbol{y}_{2}\right)\right| \leqq L\left|\boldsymbol{y}_{1}-\boldsymbol{y}_{2}\right|+\left|\int_{x_{1}}^{x_{2}} N(x) d x\right|,
$$

where $L$ is a suitable constant and $N(x)$ a non-negative summable function in $0 \leqq x \leqq a$, and moreover that for almost every point $(x, y)$ in $G$, we have

$$
\varlimsup_{t \rightarrow 0} \frac{1}{t}\{\varphi(x+t, y+t f(x, y))-\varphi(x, y)\} \leqq 0 \quad \text { a.e. . }
$$

Example. If in the equation

$$
\frac{d y}{d x}=f(x, y),
$$

$f(x, y)$ satisfies the extended Lipschitz condition, ${ }^{(9)}$ i. e., there exists a non-negeative summable function $N(x)$ such as

$$
|f(x, y)| \leqq N(x)|y|,
$$

with our theory it is enough to put

$$
\varphi(x, y)=e^{-2 I(x)} y^{2},
$$

where $L(x)=\int_{0}^{x} N(x) d x$.

\section{Mathematical Institute, Kyoto University.}

\section{Notes.}

(1) $G$ may be not necessary a bounded closed domain; for a domain such as, e. g., $a<x<b,-\infty<y_{i}<+\infty(i=1,2, \ldots, \mathrm{n})$ our discussion needs few modifications.

(2) Carathéodory; Vorlesungen über reelle Funktionen, 2te Anf., pp. 665-672.

(3) Here "inf" means "lower limit".

(4) As we shall see later, it is convenient to define like this.

(5) For $\int_{x_{P}}^{x_{Q}}\left|y^{\prime}(x)\right| d x \geqq\left|\int_{x_{P}}^{x_{Q}} y^{\prime}(x) d x\right|$.

(6) For convenience, we represent " almost everywhere " by " a. e.".

(7) Okamura; Mem. Coll. Sci. Kyoto Univ. A. 14 (1931), p.85; A 23 (1941), p.225; A.24 (1942), p.21 and Proc. of Phys-Math. Soc. of Japan. Series 3, Vol. 25 (1943), p.514.

(8) Yoshizawa and Hayashi ; Mem. Coll. Sci. Kyoto Univ. A.26 (1950), Mathematics, pp. 19-29; “Sugaku” (The Mathematics), Vol. 2, No. 4 (1950), pp. 315-318.

(9) Carathéodory; Loc. cit., p. 673. 